\title{
Identification of four genes and biological characteristics associated with acute spinal cord injury in rats integrated bioinformatics analysis
}

\author{
Qiang Li ${ }^{1,2,3 \#}$, Bo Li ${ }^{1,4 \#}$, Bo Tao ${ }^{1,2 \#}$, Chenxi Zhao ${ }^{1,2 \#}$, Baoyou Fan ${ }^{1,2}$, Qi Wang ${ }^{1,2,5}$, Chao Sun ${ }^{1,2}$, \\ Huiquan Duan ${ }^{1,2}$, Yilin Pang ${ }^{1,2}$, Xuanhao $\mathrm{Fu}^{1,2}$, Shiqing Feng ${ }^{1,2}$ \\ ${ }^{1}$ Department of Orthopedics, Tianjin Medical University General Hospital, Tianjin, China; ${ }^{2}$ International Science and Technology Cooperation \\ Base of Spinal Cord Injury, Tianjin Key Laboratory of Spine and Spinal Cord Injury, Tianjin, China; ${ }^{3}$ Department of Orthopedics, Shanxi Bethune \\ Hospital, Shanxi Academy of Medical Sciences, Taiyuan, China; ${ }^{4}$ Department of Orthopedics, Sun Yat-sen Memorial Hospital of Sun Yat-sen \\ University, Guangzhou, China; ${ }^{5}$ Department of Orthopedics, Tianjin Hospital of ITCWM Nankai Hospital, Tianjin, China \\ Contributions: (I) Conception and design: S Feng, Q Li; (II) Administrative support: S Feng; (III) Provision of study materials or patients: B Li, B Tao, \\ S Feng; (IV) Collection and assembly of data: Q Li, B Li, B Tao, C Zhao; (V) Data analysis and interpretation: Q Li, B Li; (VI) Manuscript writing: \\ All authors; (VII) Final approval of manuscript: All authors. \\ \#These authors contributed equally to this work. \\ Correspondence to: Shiqing Feng. Department of Orthopedics, Tianjin Medical University General Hospital, No. 154 Anshan Road, Heping District, \\ Tianjin 300052, China. Email: sqfeng@tmu.edu.cn.
}

Background: Spinal cord injury (SCI) is a serious condition that can cause physical disability and sensory dysfunction. Cytokines play an extremely important role in the acute phase of SCI. Clarifying the cytokine expression profile is of great importance.

Methods: Cytokine array analysis was used to explore the changes in 67 different proteins at 0 hours, 2 hours, 1 day, 3 days, and 7 days after acute SCI in rats. The differentially expressed cytokines in the various periods were analyzed and compared. The biological processes related to the differentially expressed proteins were examined using Gene Ontology (GO) analysis.

Results: Immediately after SCI (0 hours), only ciliary neurotrophic factor (CNTF) was slightly upregulated, while 23 other proteins were down-regulated. At 2 hours after SCI, there were 3 upregulated and 21 downregulated proteins. At 1 day after SCI, there were 5 upregulated and 6 downregulated proteins. At 3 days after SCI, there were 6 upregulated and 4 downregulated proteins. At 7 days after SCI, there were 4 upregulated and 9 downregulated proteins. Erythropoietin (EPO) and Fms related tyrosine kinase 3 ligand (Flt-3L) were downregulated at all time points. CD48 was decreased at 2 hours to 7 days after SCI. Monocyte chemotactic protein-1 (MCP-1) was the only protein that was upregulated at 2 hours to 7 days. The GO and pathway analyses revealed that the cytokine-related pathways, cell death, and proliferation might play a key role during secondary SCI.

Conclusions: This study identified 3 downregulated proteins during SCI, that being EPO, Flt-3L, and CD48. MCP-1 was the only upregulated protein, and its expression was upregulated till day 7 following SCI. These 4 identified genes may be potential therapeutic targets for the treatment of SCI.

Keywords: Spinal cord injury (SCI); inflammation; erythropoietin; Fms related tyrosine kinase 3 ligand (Flt-3L); CD48; monocyte chemoattractant protein-1 (MCP-1)

Submitted Jan 11, 2021. Accepted for publication Mar 05, 2021.

doi: $10.21037 / \mathrm{atm}-21-603$

View this article at: http://dx.doi.org/10.21037/atm-21-603 


\section{Introduction}

Spinal cord injury (SCI) is a traumatic disease that causes exercise, sensory, or autonomic dysfunction, thereby directly affecting the quality of life of patients and imposing a heavy financial burden on society and families (1). It is therefore crucial to clarify the pathophysiological processes associated with SCI. After SCI, secondary injury such as inflammation, oxidative stress, and apoptosis, can lead to even more serious consequences. Ultimately, secondary injury induces cell death, demyelination, and axonal degeneration at the epicenter of the injury and the surrounding regions $(2,3)$. Inflammatory reactions mediated by cytokines and other factors plays an important role in the development of secondary injury following SCI $(4,5)$. Inflammatory factors are important bridges between immune cells and other cells and are important mediators in regulating the immune response. These cytokines include pro-inflammatory factors, anti-inflammatory factors, chemokines, and neurotrophic factors. Studies have shown that the critical balance between these processes plays an important role in the progress and outcome of the neurodegenerative process (5-7).

The role of inflammation in the process of SCI is complex, with certain beneficial aspects as well, such as the removal of cellular debris, a number of studies have suggested that inflammatory responses spread the damage to surrounding tissue, induce apoptotic cell death, and impair spontaneous regeneration and functional recovery (3). The infiltration of leukocytes and activation of glial cells can aggravate tissue damage by releasing proteases, reactive oxygen intermediates, lysosomal enzymes, and inflammatory cytokines (8). Studies found that cytokines may be sensitive markers of disease. Cytokines network, but not a single cytokine, responses to environmental hazards. High-throughput detection of cytokines provides a global view of changes cytokine profiles and helps us identify many vital differentially expressed cytokines in the development of SCI. Inflammatory factors include those that are pro-inflammatory and those that are antiinflammatory. Pro-inflammatory factors such as interleukin (IL)-1 $\beta$, IL-6, and tumor necrosis factor (TNF)- $\alpha$ play a vital role in the secondary injury of SCI, and blockers or knockout of related genes improve spinal cord function (9-13). Studies have also shown that IL-6 facilitates spinal cord repair (14). Anti-inflammatory factors, such as granulocyte-macrophage colony-stimulating factor (GMCSF), IL-10, and IL-13, promote the recovery of nerve function $(5,15)$. Erythropoietin (EPO), the erythropoietic hormone is a leading therapy in neonatology as a neuroprotective agent (16). Monocyte chemoattractant protein-1 (MCP-1) is a member of the chemotactic cytokine family that functions to recruit monocytes and modulate T-cell function (17). Fms related tyrosine kinase 3 ligand (Flt-3L) causes dendritic cell and lymphocyte proliferation (18). CD48, a member of the signaling lymphocyte activation molecule family, participates in adhesion and activation of immune cells (19). At different time points after SCI, the cytokine expression trends are different, indicating that different key cytokines appear at different time points. Targeting key cytokines at precise time points may exert maximum treatment efficacy. Thus, clarifying the cytokine expression profiles at different times following SCI will benefit the understanding of the underlying mechanisms and aid in the development of effective therapeutic strategies.

While previous studies have focused on several major inflammatory factors, the expression of cytokines after SCI has not been fully analyzed. Jiang et al. identified 5 hub genes with high-throughput sequencing after compression SCI (20). In this study, we established a weight-drop SCI model, it has advantages of simple performance, good controllability and repeatability compare with compression SCI model. As the protein expression of genes is the results of transcription and translation. The protein expression could be regulated by miRNA, IncRNA and circRNA at post-transcription level. Proteins is the main undertaker of life activities. Thus, we chose to use protein array technology to compare and analyze the expression of various cytokines, rather than mRNA. The protein array technology is more conductive to reflect the occurrence and the development of SCI. These results will provide new sights into the underlying mechanisms and contribute to the design of novel therapeutic methods. We present the following article in accordance with the ARRIVE reporting checklist (available at http://dx.doi.org/10.21037/atm-21603).

\section{Methods}

\section{Ethics statement and animal grouping}

A total of thirty 10 -week-old female Wistar rats $(250 \pm 25 \mathrm{~g})$ were purchased from the Tianjin Institution of Radiation Medicine. The rats were housed under continuous humidity conditions in 12-hour day/night cycles. Rats were randomly separated into two groups, with 5 rats in the sham group and 25 rats in the injury group. Spinal cord tissue was collected immediately after SCI (0 hour) and at 4 other time points, 
namely, 2 hours, 1 day, 3 days, and 7 days (5 rats/time point). All experimental procedures were approved by the Ethics Committee of Tianjin Institute of Radiation Medicine (approval number: IRM-DWLL-2019039) and were performed according to the National Institutes of Health in the Guide for the Care and Use of Laboratory Animals (NIH Publications no. 85-23, revised 1996).

\section{Establishing the SCI model}

The SCI model was established using the New York University Impactor device (NYU, New York, NY, USA) as described previously (21). Briefly, rats were anesthetized with chloral hydrate $(4 \%, 3 \mathrm{~mL} / \mathrm{kg})$ and the spinal cord was exposed after T10 laminectomy. The spinal cord was confused with a $10 \mathrm{~g} \times 25 \mathrm{~mm}$ free dropped node. When the spinal cord was injured by the node, the tail was twisted and the hindlimbs were shaken immediately. The incision was then sutured, and the rats were kept on a warm pad until they regained consciousness. The spinal cord samples were extracted from $5 \mathrm{~mm}$ around the epicenter of damage at the indicated times.

\section{Protein array detection}

The proteins in the spinal cord samples were extracted and detected using the Rat Cytokine Array 67 (Raybiotech, Norcross, GA) according to the manufacturer's instructions. Briefly, the proteins in spinal cord samples were extracted with cell lysis buffer and a protease inhibitor cocktail (Raybiotech, Norcross, GA). After determining the protein concertation of the samples with the BCA protein assay kit (Raybiotech, Norcross, GA), the samples were incubated with protein array glass slides. A $100 \mu \mathrm{L}$ sample $(500 \mu \mathrm{g} / \mathrm{mL})$ was added to each cell of the glass slide and incubated at $4{ }^{\circ} \mathrm{C}$ overnight. The slides were then washed with Thermo Scientific Well Wash Versa (Thermo Scientific, USA) and incubated with a biotinylated antibody cocktail and $\mathrm{Cy} 3$ equivalent Dye-Streptavidin. Thereafter, the signals were visualized using a laser scanner. Serial dilutions of a predetermined calibration standard mix were used to generate a standard curve for each cytokine. The cytokine concentrations in the samples were calculated according to the standard curve.

\section{Gene Ontology (GO) analyses}

The biological processes related to the differentially expressed proteins (DEPs) were investigated using GO analysis, the Fisher test, and cluster Profiler data package from R/Bioconductor. When more than 5 differentially expressed proteins were identified in a particular GO term and $\mathrm{P}<0.05$, the results were considered significant.

\section{Statistical analysis}

Experimental data were analyzed using SPSS 20.0 software and expressed as the mean \pm standard error (SD). The statistical differences in protein expression between two groups were evaluated by Student's unpaired $t$-test. For more than two group, statistical significance was determined with one-way analysis of variance (ANOVA) test, followed by Dunnett's LSD post hoc testing to calculate the longitudinal differences between groups. A value of $\mathrm{P}<0.05$ was considered statistically significant.

\section{Results}

\section{Hierarchical clustering was used to analyze the profiles of the differentially expressed proteins at different time points after $S C I$}

To identify the differentially expressed proteins at 0 hour, 2 hours, 1 day, 3 days, and 7 days after SCI, hierarchical clustering was conducted. As showed in Figure $1 \mathrm{~A}$, there were more down-regulated proteins at the 0 -hour and 2 -hour time points, and more up-regulated proteins at 1,3 , and 7 days after SCI. These 67 proteins were divided into 3 clusters, namely a high expression group $(>1,000 \mathrm{pg} / \mathrm{mL}$ ), a moderate expression group (expression levels between 100 and $1,000 \mathrm{pg} / \mathrm{mL}$ ), and a low expression group $(\leq 100 \mathrm{pg} / \mathrm{mL})$. Among the 67 proteins, at all timepoints low expression proteins were the most abundant, moderate expression proteins ranked the second, while high expression proteins were the least abundant. At the 0 -and 2-hour time points, the number of proteins in the moderate cluster decreased and the number of proteins in the low expression cluster increased (Figure 1B). The proteins that sustained its expression in the same cluster at all time points were shown in Figure 1C. The moderate expression cluster had more proteins than the other two clusters.

\section{The protein expression patterns following SCI}

A total of 6 different expression patterns were observed for the 67 differentially expressed proteins and these have 

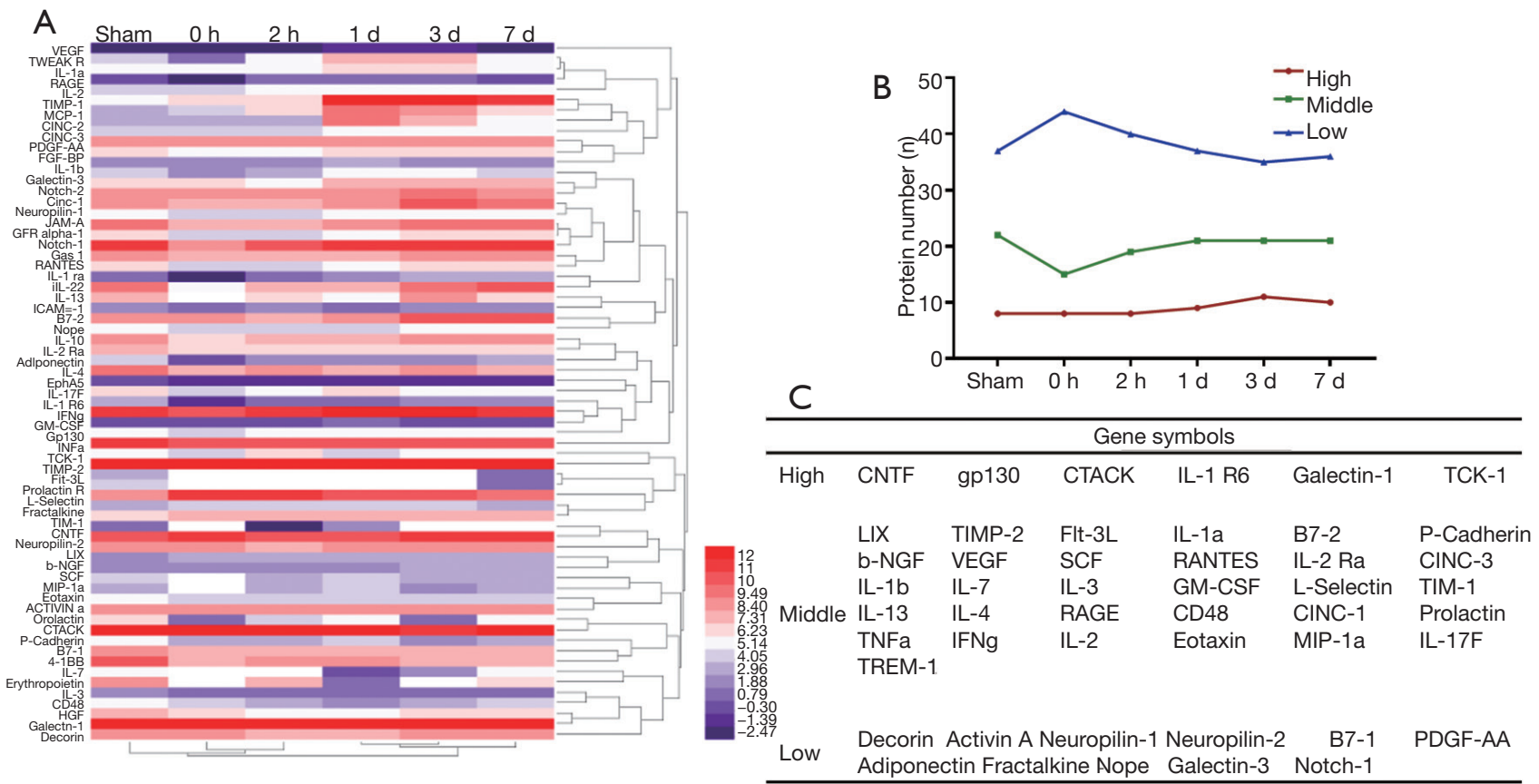

Figure 1 The profiles of the differentially expressed proteins at different time points following spinal cord injury. (A) The heat-map shows the upregulated genes (red) and downregulated genes (blue) between the sham and injury groups at 0 hours, 2 hours, 1 day, 3 days, and 7 days after SCI ( $n=5$ in the injury group at all time points except day $3 ; n=4$ in the injury group at day $3 ; n=11$ in the sham group). (B) The distribution of proteins in the high, moderate, and low expression clusters. (C) The proteins within the same cluster with sustained expression throughout all time points. SCI, spinal cord injury; CNTF, ciliary neurotrophic factor; gp130, Glycoprotein 130; CTACK, human chemokine (C-C Motif) ligand 27; TCK-1, thymus chemokine-1; LIX, LPS-induced CXC chemokine; TIMP-2, tissue inhibitor of metalloproteinases-2; F1t-3L, Fms related tyrosine kinase 3 ligand; IL-1a, interleukin-1Alpha; B7-2, cluster differentiation 86; P-Cadherin, placental cadherin; b-NGF, beta-nerve growth factor; VEGF, vascular endothelial growth factor; SCF, Stem cell factor; RANTES, regulated upon activation normal T cell expressed and secreted factor; IL-2 Ra, interleukin-2 receptor; CINC-3, cytokine induced neutrophil chemoattractant-3; IL-1b, interleukin-1 beta; IL-7, interleukin-7; IL-3, interleukin-3; GM-CSF, Granulocyte-macrophage colony stimulating factor; L-Selectin, leucocyte-selectin; TIMP-1, tissue inhibitor of metalloproteinases-1; IL-13, interleukin-13; IL-4, interleukin-4; RAGE, receptor for advanced glycosylation end products; CD48, cluster differentiation 48; CINC-1, cytokine induced neutrophil chemoattractant-1; TNF- $\alpha$, tumor necrosis factor- $\alpha$; IFNg, Interferon Gamma; IL-2, interleukin-2; MIP-1a, macrophage inflammatory protein-1a; IL-17F, interleukin-17F; TREM-1, triggering receptor expressed on monocytes-1.

been denoted as follows: decrease (D pattern, observed in 12 proteins, Figure 2), decrease-increase (D-I pattern, observed in 19 proteins, Figure 3), decrease-increasedecrease (D-I-D pattern, observed in 24 proteins, Figure 4), increase (In pattern, observed in 2 proteins, Figure 5, upper row), increase-decrease (In-D pattern, observed in 8 proteins, Figure 6). and irregularity (Ir pattern, observed in 3 proteins, Figure 5, lower row). Among the 6 patterns, the D-I-D, D-I, and D patterns were the most abundant, indicating that decreased expression was the main trend for most of the differentially expressed proteins.

\section{The differentially expressed proteins at different time points after SCI}

To better demonstrate the differentially expressed proteins at different time points, volcano-plot analyses were conducted. The proteins were taken into consideration when the fold change (FC) was over 1.2 or less than 0.83 . At 0 hours after SCI, only ciliary neurotrophic factor (CNTF) was slightly up-regulated, while 23 proteins were down-regulated, with EPO being the most downregulated. At 2 hours after SCI, there were 3 upregulated and 21 downregulated proteins. MCP-1 was the most upregulated and EPO was the most down-regulated protein. At 1 day 

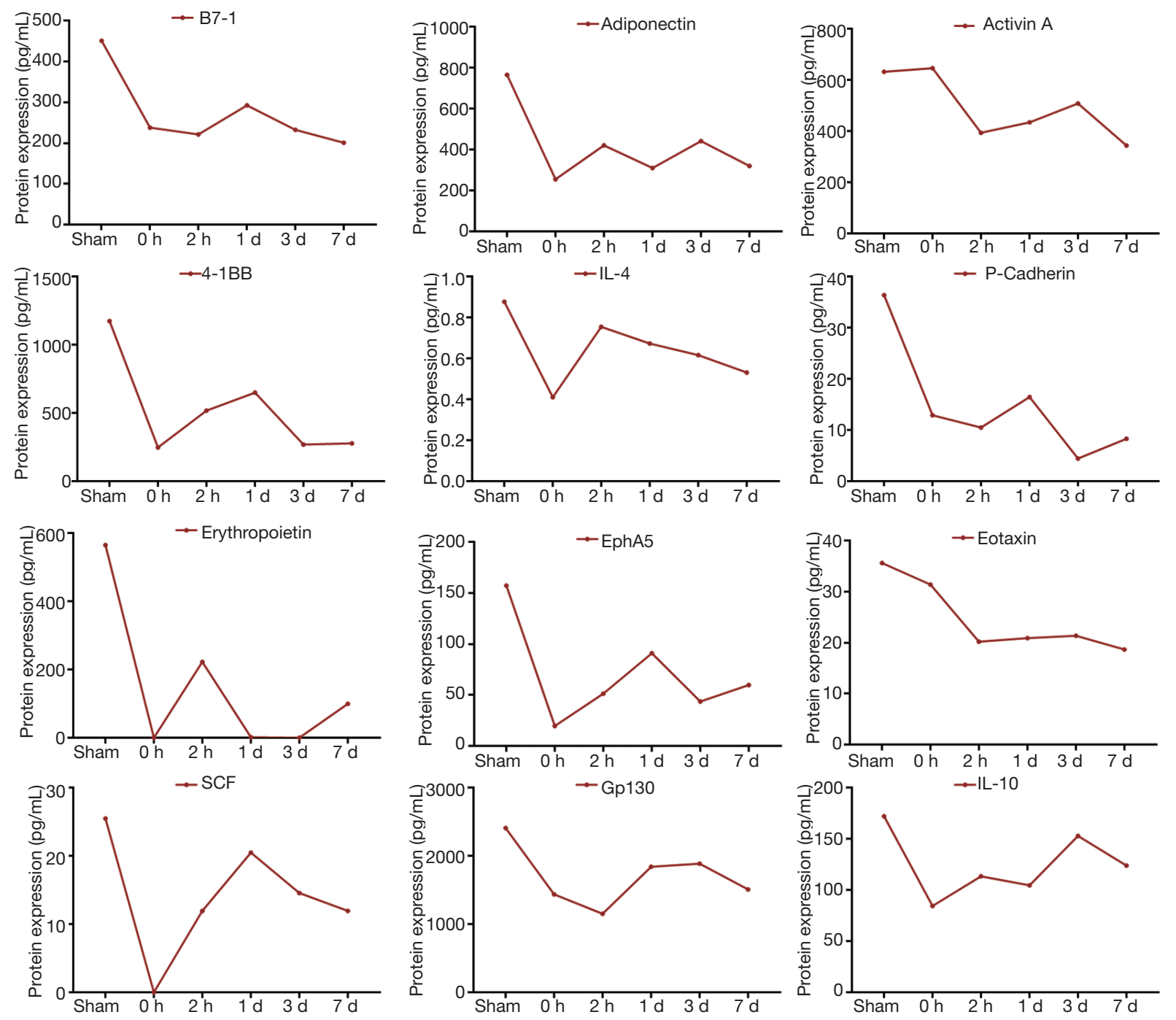

Figure 2 The differentially expressed proteins with a decreased pattern of expression. Protein expression analysis revealed 12 proteins with a decreased expression trend in the injury group at 0 hours, 2 hours, 1 day, 3 days, and 7 days after SCI compared to the sham group. SCI, spinal cord injury; B7-1, cluster differentiation 80; 4-1BB, cluster differentiation137; IL-4, interleukin-4; SCF, stem cell factor.

after SCI, there were 5 upregulated and 6 downregulated proteins. At 3 days after SCI, there were 6 upregulated and 4 downregulated proteins. At 7 days after SCI, there were 4 upregulated and 9 downregulated proteins. Tissue inhibitor of metalloproteinases-1 (TIMP-1) and MCP-1 were the 2 most upregulated proteins and EPO was still the most downregulated protein at day 1 , day 3 , and day 7 (Figure $7 A, B, C, D, E)$.

\section{Analysis of the key proteins in the acute phase of SCI}

To determine which key proteins play a pivotal role in the acute phase of SCI, Venn plots were used to show the differentially expressed proteins at more than three time points (Figure 8A,B). EPO and c were differentially expressed at all time points. CD48 and MCP-1 were differentially expressed from 2 hours to 7 days. Nope and Notch-1 were differentially expressed from 0 hours to 1 day, while TIMP-1 was differentially expressed from 1 to 7 days. The expression profiles of EPO, Flt-3L, CD48, and MCP-1 at all time points are shown in Figure 8C,D. MCP-1 was the only protein that was upregulated at all time points, while Flt-3L, CD48, and EPO were downregulated at all time points. The latter 3 proteins had similar expression 

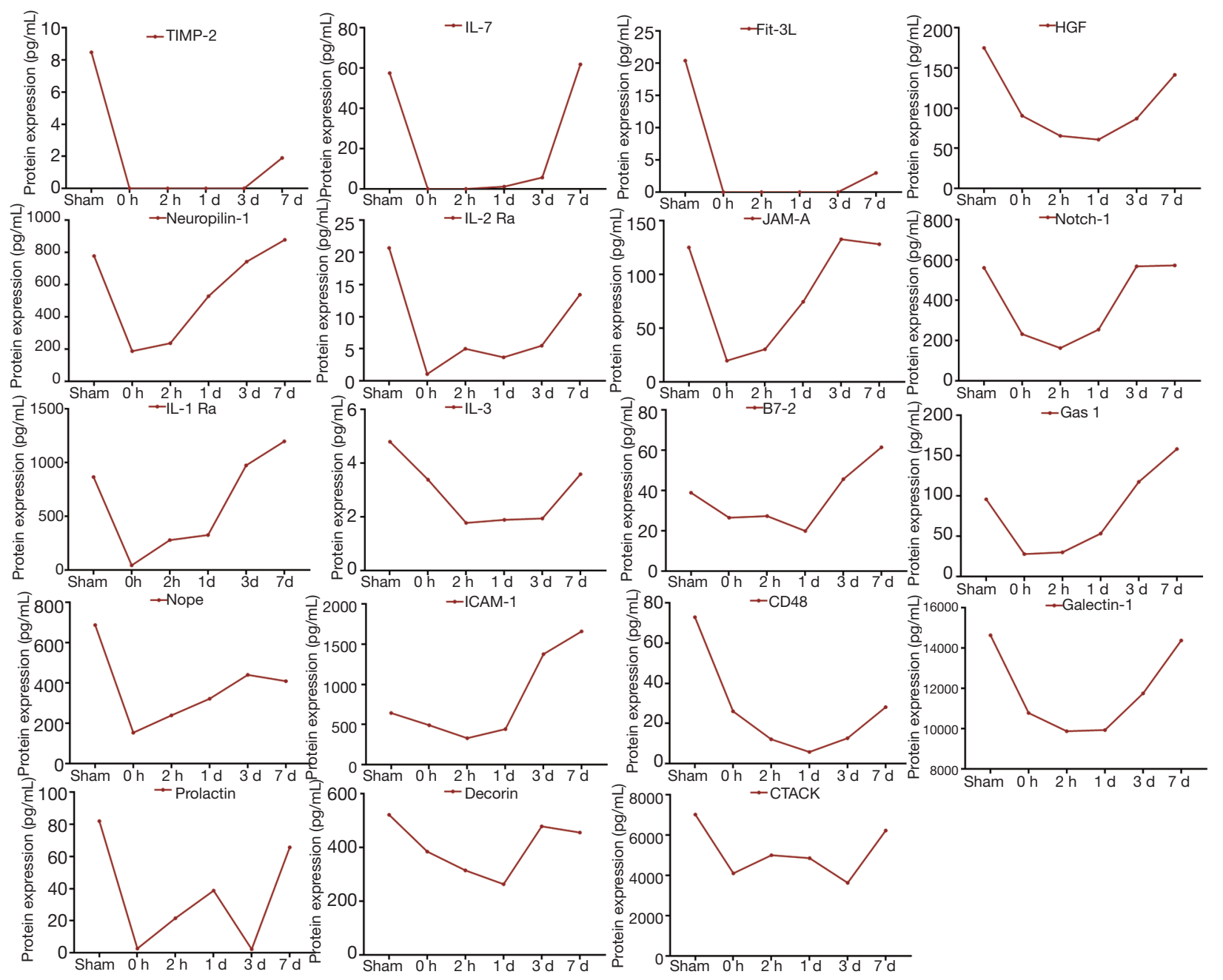

Figure 3 The differentially expressed proteins showing a decrease-increase pattern. Protein expression analysis identified 19 proteins with a decrease-increase expression trend in the injury group at 0 hours, 2 hours, 1 day, 3 days, and 7 days after SCI compared to the sham group. SCI, spinal cord injury; TIMP-2, tissue inhibitor of metalloproteinases-2; IL-7, interleukin-7; F1t-3L, Fms related tyrosine kinase 3 ligand; JAM-A, junction adhesion molecule-A; CTACK, human chemokine (C-C Motif) ligand 27.

patterns. Flt-3L was expressed at a low level in normal rats but was not detectable in SCI rats at 0 hours to 3 days, followed by a slightly increased expression at 7 days. EPO was also the most downregulated protein over the course of the experiment.

\section{The gene ontology and pathway analysis of the differentially expressed proteins}

To better clarify the biological processes of the DEPs at different time points after SCI, GO and pathway analyses were performed. "Signaling-receptor binding" and "molecular function regulators" were the 2 most enriched
GO molecular functions detected. "Cell surface" and "intrinsic component of plasma membrane" were the top 2 enriched GO cellular components detected. The top 10 GO biological processes related to the differentially expressed proteins were "regulation of cell death", "regulation of cell proliferation", "defense response", "regulation of programmed cell death", "regulation of apoptotic process", "response to cytokine", "cellular response to cytokine stimulus", "positive regulation of cell proliferation", "cytokine-mediated signaling pathway", and "response to wounding" (Figure 9). The top 4 pathways were "cytokine signaling in immune system", "cytokine-cytokine receptor interaction", "extracellular matrix (ECM) and associated 


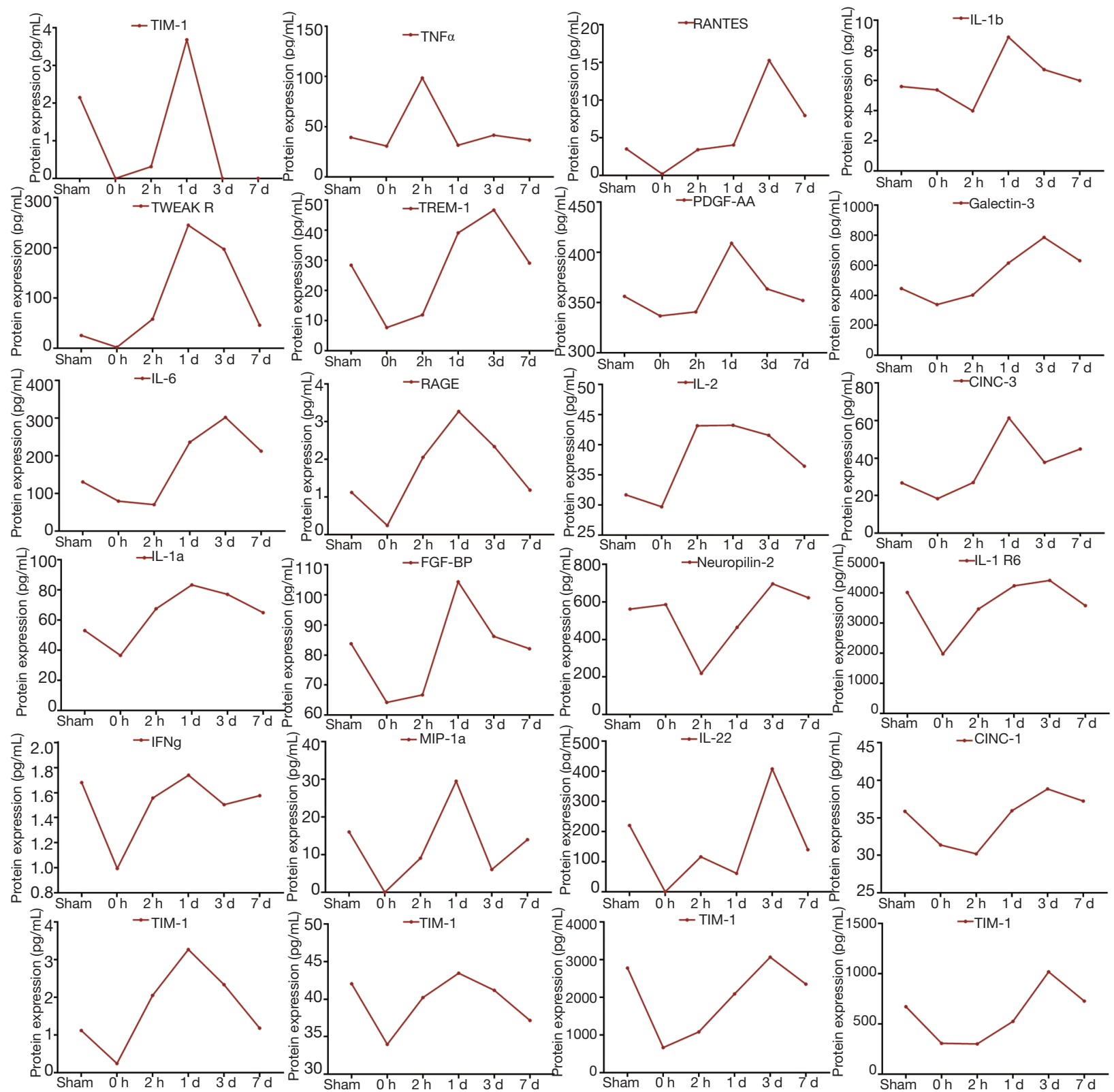

Figure 4 The differentially expressed proteins with a decrease-increase-decrease pattern. Protein expression analysis identified 24 proteins with a decreased-increase- decreased expression trend in the injury group at 0 hours, 2 hours, 1 day, 3 days, and 7 days after SCI compared to the sham group. SCI, spinal cord injury; TIM-1, tissue inhibitor of metalloproteinases-1; TNF- $\alpha$, tumor necrosis factor- $\alpha$; RANTES, regulated upon activation normal $\mathrm{T}$ cell expressed and selected factor; TWEAK R, tumor necrosis factor receptor superfamily member $12 \mathrm{~A}$; PDGF-AA, platelet-derived growth factor-AA; RAGE, receptor for advanced glycosylation end products; FGF-BP, fibroblast growth factor binding protein; CINC-2, cytokine induced neutrophil chemoattractant-2; GM-CSF, granulocyte-macrophage colony stimulating factor; GFR alpha-1, GDNF family receptor alpha-1. 


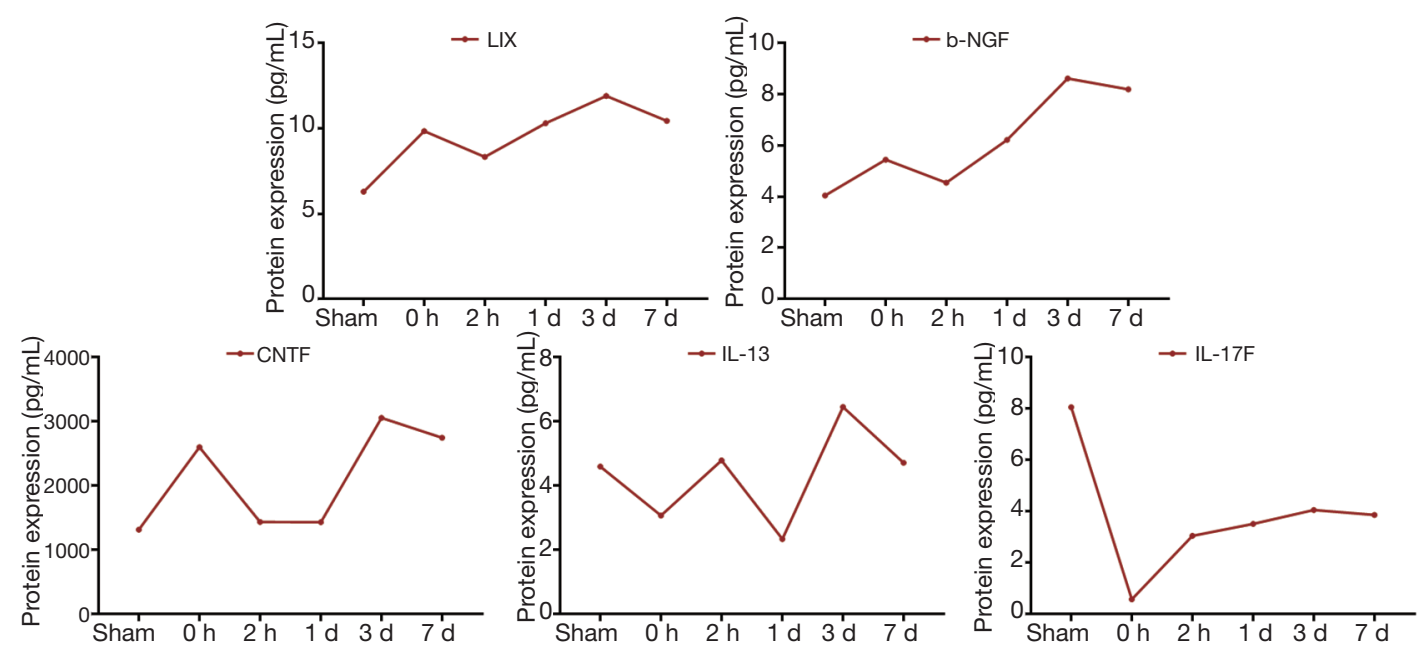

Figure 5 The differentially expressed proteins with an increased pattern (upper row) and an irregular pattern (lower row). Protein expression analysis showed 2 proteins with an increased expression trend and 3 proteins with an irregular expression trend in the injury group at 0 hours, 2 hours, 1 day, 3 days, and 7 days after SCI compared to the sham group. SCI, spinal cord injury; LIX, LPS-induced CXC chemokine; b-NGF, beta-Nerve Growth Factor; CNTF, ciliary neurotrophic factor; IL-13, interleukin-13; IL-17F, interleukin-17F.
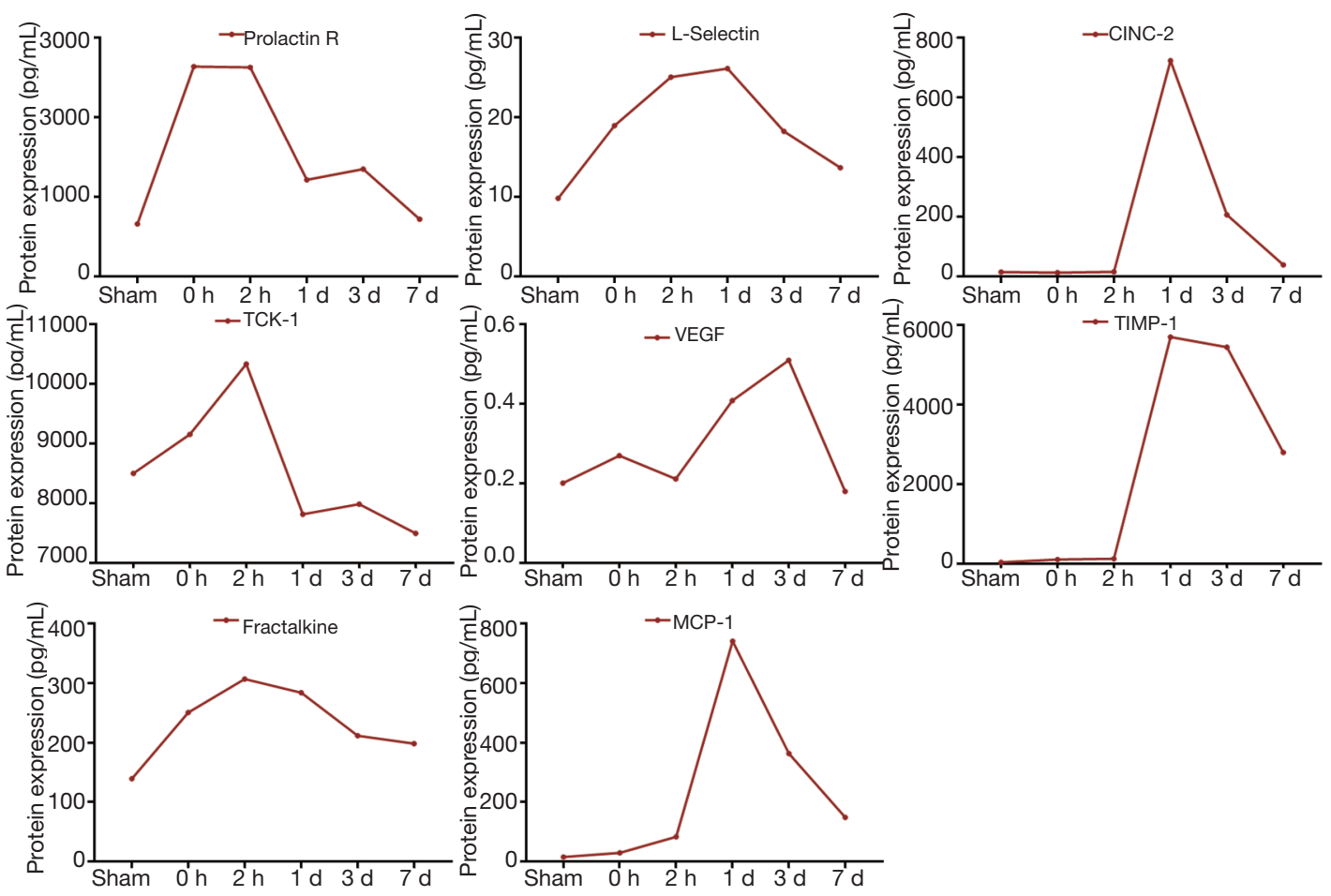

Figure 6 The differentially expressed proteins with an increase-decrease pattern. Protein expression analysis showed 8 proteins with an increase-decrease expression trend in the injury group at 0 hours, 2 hours, 1 day, 3 days, and 7 days after SCI compared to the sham group. SCI, spinal cord injury; CINC-2, cytokine induced neutrophil chemoattractant-2; TCK-1, Tilletia controversa Kühn-1; VEGF, vascular endothelial growth factor; TIMP-1, tissue inhibitor of metalloproteinases-1; MCP-1, monocyte chemoattractant protein-1. 

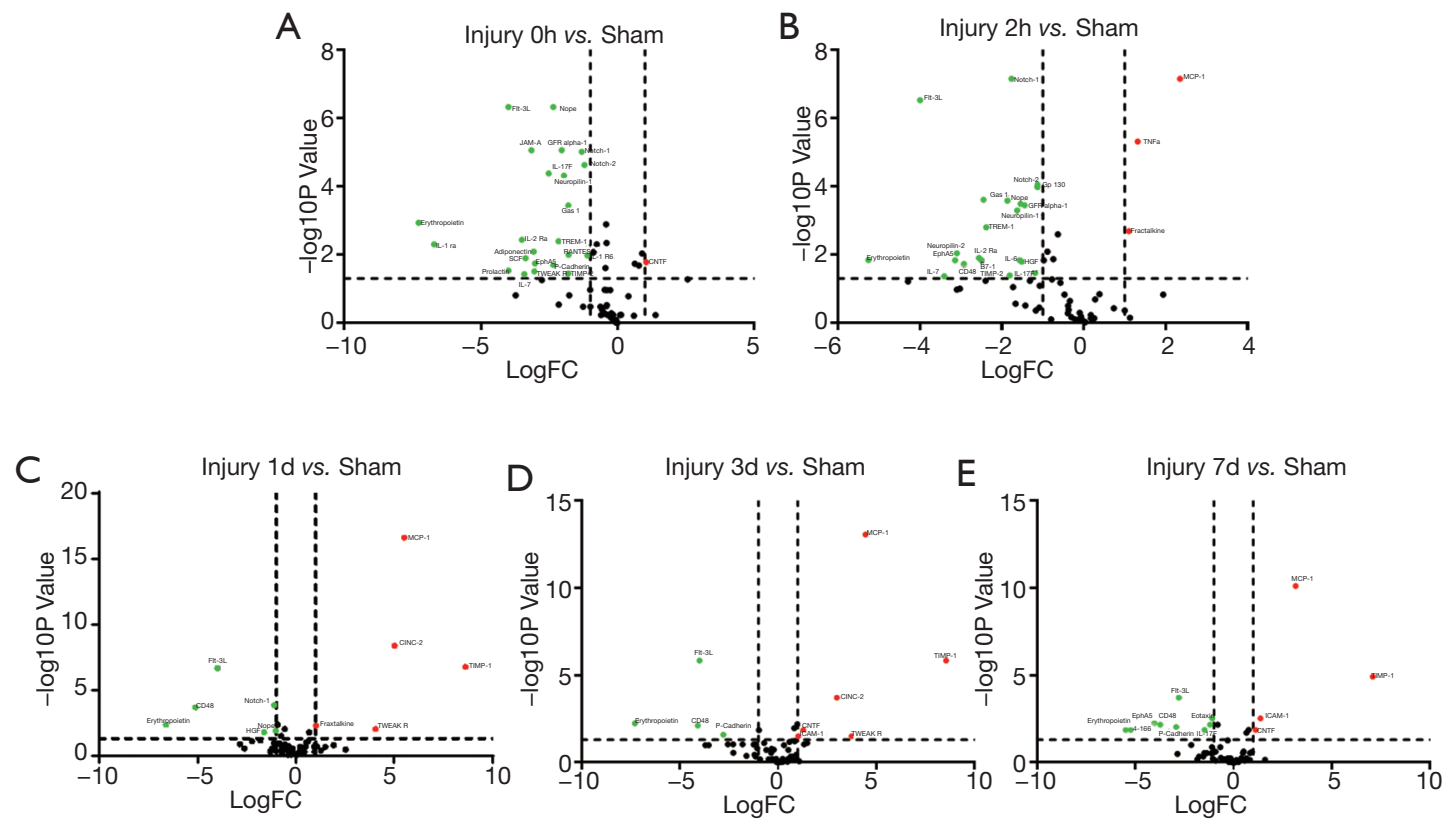

Figure 7 Volcano-plot showing the differentially expressed proteins at different time points after spinal cord injury (SCI). (A-E) The volcano-plot shows upregulated genes (red) and downregulated genes (green) in the sham group and the injury group at 0 hours, 2 hours, 1 day, 3 days, and 7 days after SCI. The $\mathrm{X}$-axis represents the $\log \mathrm{FC}$ (fold change), and the two dotted lines at the $\mathrm{X}$-axis are at $\mathrm{X}=1$ and $\mathrm{X}=-1$ (presenting 2-fold upregulation and 2-fold downregulation, respectively). The Y-axis represents the $-\log 10 \mathrm{P}$ value, and the dotted line at the $\mathrm{Y}$-axis is at $\mathrm{Y}=1.30103(\mathrm{P}=0.05)$. The upper right red dots represent the differentially expressed proteins with $\mathrm{FC}>2$ and $\mathrm{P}<0.05$, and the upper left green dots represent the differentially expressed proteins with $\mathrm{FC}<2$ and $\mathrm{P}<0.05$.

proteins", and "regulators and secreted factors". These results indicated that cytokine-related pathways, cell death, and proliferation may play a key role during secondary SCI.

\section{Discussion}

SCI results in traumatic injury to the central nervous system leading to locomotor and sensory function loss. Novel therapeutic strategies such as anti-inflammatory targets, anti-axon growth inhibitory molecules, stem cell transplantation, and biomaterial transplantation have been developed in animal studies and clinical trials. However, to date, there are no effective treatment strategies to promote recovery of spinal cord functional in the clinical setting. The mechanisms of secondary SCI are still not fully understood and this presents a significant barrier to developing effective treatment strategies. Thus, unravelling the precise mechanisms underlying SCI is of great importance to the development of effective treatment strategies.

SCI is divided into the super acute phase, the acute phase, the subacute phase, and the chronic phase depending on time since injury. The super acute phase is the minutes immediately following SCI, and the acute phase is one week after SCI. The inflammatory reaction, which is the main mechanism of acute SCI $(13,22,23)$, contributes greatly to secondary SCI and is a complex process that involves invading immune cells, such as neutrophils and macrophages, and astrocyte and microglia (23). The immune system is a major contributor to the pathogenesis of spinal cord injury. T and B lymphocytes that respond to the same antigen migrate to the spinal cord injury site, resulting in a multifaceted adaptive immune response (24). Lymphocyte's infiltration occurs in the first week after SCI and maintained for a long time. In genetic mice which lack T-cells could find functional and tissue improvement and B-cell knockout mice have improved BMS scores associated with decreased lesion following SCI (25). This observation may be due to an enhanced regenerative response in the absence of lymphocytes, or a reduction in immunemediated tissue injury when $\mathrm{T}$ - and B-cells are removed. The cytokines secreted from immune cells, astrocyte, and microglia are rapidly expressed within minutes after SCI (26). Investigating the cytokine expression profile after SCI is of great importance in elucidating the underlying 


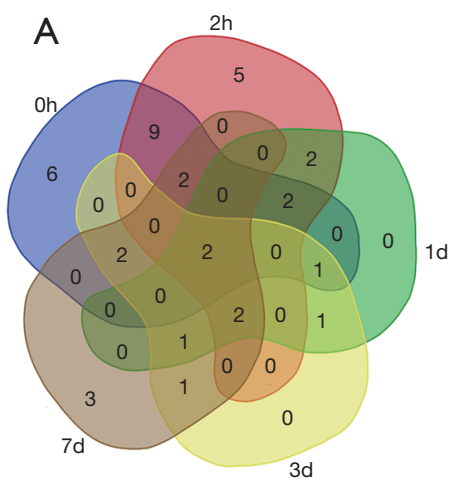

B

\begin{tabular}{lll}
\hline Names & Total & Proteins \\
\hline Oh $2 \mathrm{~h}$ 1d 3d 7d & 2 & Erythropoietin Flt-3L \\
2h $1 \mathrm{~d}$ 3d $7 \mathrm{~d}$ & 2 & CD48 MCP-1 \\
Oh $2 \mathrm{~h}$ 1d & 2 & Nope Notch-1 \\
Oh $2 \mathrm{~h}$ 7d & 2 & EphA5 IL-17F \\
Oh $1 \mathrm{~d}$ 3d & 1 & TWEAK R \\
Oh 3d $7 \mathrm{~d}$ & 2 & CNTF P-Cadherin \\
1d 3d 7d & 1 & TIMP-1
\end{tabular}
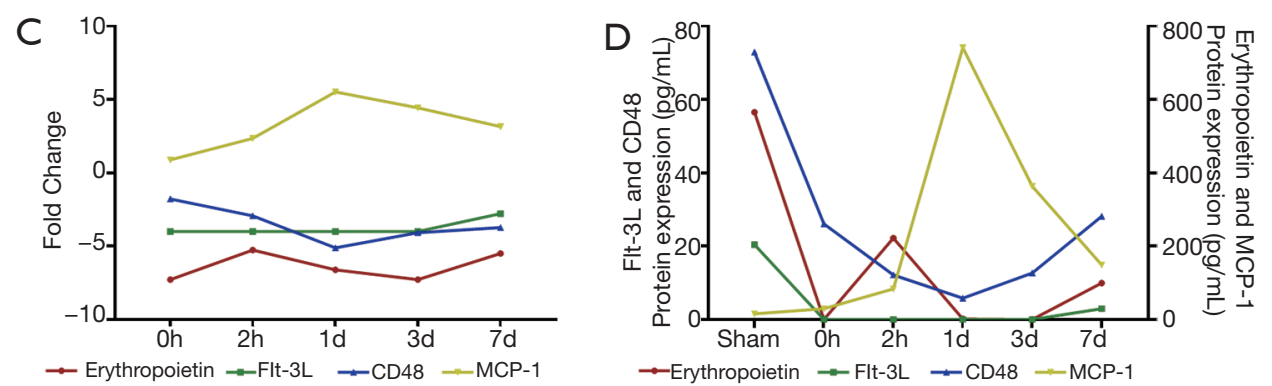

Figure 8 An analysis of the key proteins in the acute phase of spinal cord injury. (A) The Venn diagram shows co-dysregulated proteins at 0 hours, 2 hours, 1 day, 3 days, and 7 days after SCI. (B) The proteins that are co-dysregulated at more than 3 time points are shown. (C,D) The expression profiles of the proteins that are co-dysregulated at more than 4 time points are shown. SCI, spinal cord injury; Flt-3L, Fms related tyrosine kinase 3 ligand; MCP-1, monocyte chemoattractant protein-1; IL-17F, interleukin-17F; TWEAK R, Tumor necrosis factor receptor superfamily member 12A; CNTF, ciliary neurotrophic factor; TIMP-1, tissue inhibitor of metalloproteinases-1.

pathophysiological processes of SCI. Unlike polymerase chain reactions (PCRs), enzyme linked immunoassays (ELISAs), or Western blot analyses, which can only detect one marker one time (27), cytokine array is a sensitive, high specificity technique that only requires a small sample and can detect a multitude of proteins (up to 500 markers). Hence, this is an effective method to study protein expression profiles following SCI (28).

Some studies have simultaneously detected multiple protein expressions after SCI. Lee et al. detected 9 differentially expressed proteins using Real-time quantitative polymerase chain reaction (RT-qPCR) and immunohistochemistry within 21 days post-SCI. TNF- $\alpha$ mRNA was upregulated 1 hour post-injury and decreased to baseline by 3 days post-injury. MCP- 1 and macrophage inflammatory protein (MIP)-1 $\alpha$ were also detected after SCI. MCP-1 mRNA increased 1 hour after SCI and decreased to baseline after 14 days (4). Hong et al. detected levelspecific differences in vascular endothelial growth factor (VEGF), leptin, and IL10 expression in SCI rat plasma used high-throughput ELISA panels. Another study detected 6 differentially expressed cytokines in the serum of patients with chronic SCI ( $>12$ months), with both IL-2 and TNF- $\alpha$ levels upregulated (29). In our study, 67 differentially expressed proteins were detected and analyzed, which is a much larger panel of proteins identified compared to previous studies. The upregulated proteins such as the proinflammatory factors, were most likely derived from the activated immune cells in the blood or leaked proteins due to damage in the blood spinal cord barrier. The differentially expressed proteins in spinal cord tissues at different time points could explain the pathophysiological processes after injury. Thus, detecting the differentially expressed proteins in the injured spinal cord at different time points is of great importance to clarify the events in SCI.

In this study, the Rat Cytokine Array 67 was used to demonstrate the shifts in cytokine expression at 0 hours, 2 hours, 1 day, 3 days, and 7 days after SCI. The 67 cytokines were separated into high, moderate, and low expression clusters according to their expression level. The low expression cluster had the most proteins, while the moderate expression cluster had the most stably expressed 


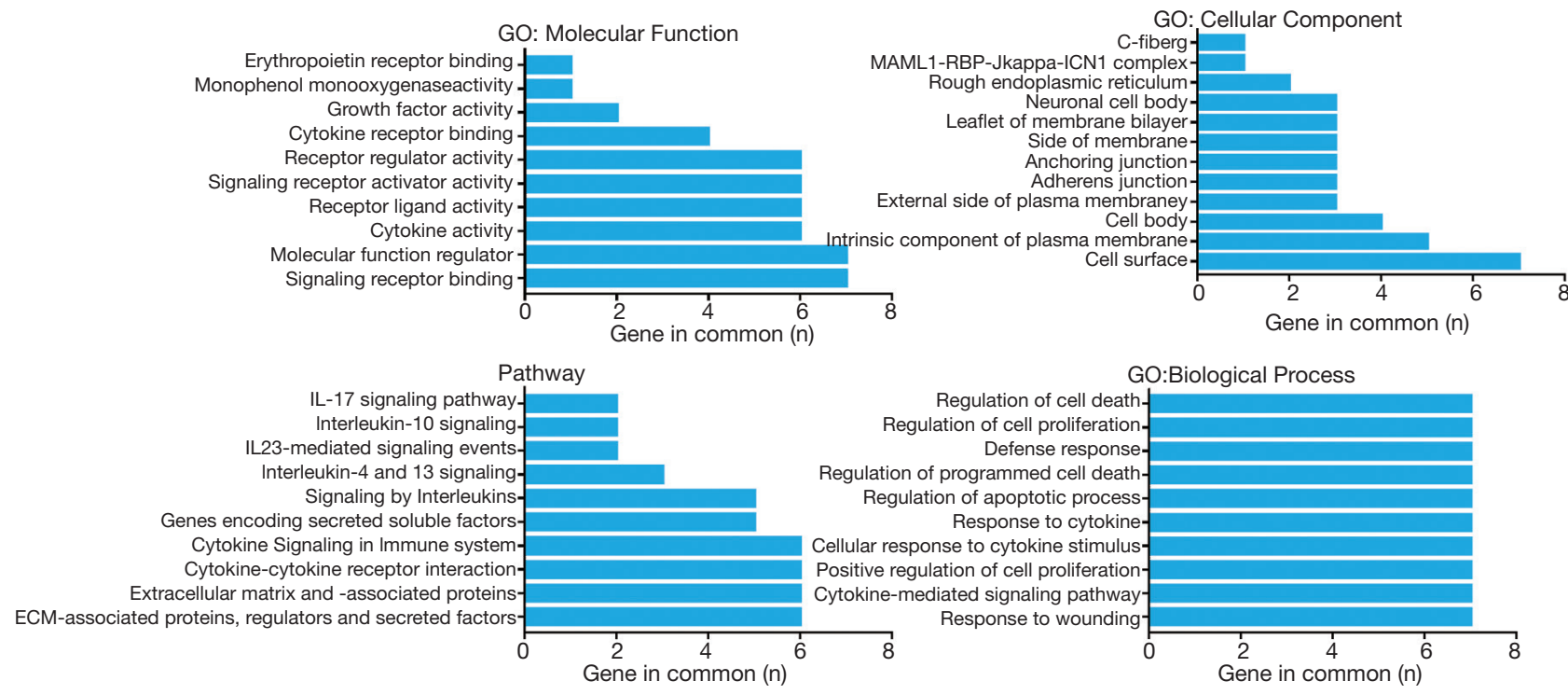

Figure 9 The Gene Ontology (GO) and pathway analysis of the differentially expressed proteins. The ordinate represents the GO and pathway terms, and the abscissa represents the number of genes related to the GO and pathway terms.

proteins at all time points. There were 6 protein expression patterns among the 67 proteins. The 3 most common patterns according to protein numbers were decreaseincrease-decrease, decrease-increase, and decrease. There were 4 proteins with expression changes at more than 4 time points with statistical significance. Of these 4 proteins, EPO, Flt-3L, and CD48, were downregulated till 7 days after SCI, and MCP-1 was the only upregulated protein at all time points. The differential expression of these 4 proteins suggested that they may have a regulatory role in secondary SCI. EPO may protect tissues via its antioxidation, anti-apoptosis, and neuroprotection abilities. It protects the spinal cord from ischemia and reperfusion injury (30). In fact, EPO has been developed as a therapeutic drug to protect against cell death (7). In our study, EPO was normally expressed in rats given a sham operation, but its downregulation was sustained in rats following SCI. The downregulated EPO expression indicated that the injured spinal cord could not resist secondary injury. Thus, upregulation of EPO as soon as possible after SCI may be beneficial for functional recovery of the injured spinal cord. Flt-3L is one of the growth factors that is significantly decreased in stroke patients (6). Application of Flt-3L decreases the amount of latent virus in infected neurons (31). The downregulation of Flt-3L in our study also suggested poor anti-injury and regenerative ability. CD48 is thought to be a receptor expressed on hematopoietic cells and serves as an adhesion and costimulatory molecule (32). In our result, CD48 was expressed normally in sham rats, but its expression decreased during the acute phase of SCI. This suggested that $\mathrm{CD} 48$ may have a potential regulatory role and may act as a beneficial receptor in SCI. Consistent with other studies, MCP-1 was upregulated after SCI (33). The expression of MCP-1 can be induced by many inflammatory factors such as IL- $1 \beta$ and TNF- $\alpha$ (33). It is a chemokine that binds to the $\mathrm{CC}$ chemokine receptor- 2 of inflammatory cells. Reports have demonstrated that anti-MCP-1 antibodies have a beneficial effect on tissue protection following SCI (33). Thus, inhibiting MCP-1 expression at the early stages of SCI may be an effective method for the treatment of SCI.

\section{Conclusions}

In conclusion, this study identified 3 downregulated proteins, EPO, Flt-3L, and CD48, and 1 upregulated protein, MCP-1 that remained upregulated until day 7 following SCI. Regulation of these 4 proteins, separately or together, at the early stages of SCI may be a novel strategy for the repair of SCI.

\section{Acknowledgments}

Funding: This work was supported by the Key Projects for Science and Technology Support (19YFZCSY00660), Tianjin Research Innovation Project for Postgraduate 
Students (2019YJSB106) and Key Projects of Tianjin Natural Science Foundation (19JCZDJC34900).

\section{Footnote}

Reporting Checklist: The authors have completed the STROBE reporting checklist. Available at http://dx.doi. org/10.21037/atm-21-603

Data Sharing Statement: Available at http://dx.doi. org/10.21037/atm-21-603

Conflicts of Interest: All authors have completed the ICMJE uniform disclosure form (available at http://dx.doi. org/10.21037/atm-21-603). The authors have no conflicts of interest to declare.

Ethical Statement: The authors are accountable for all aspects of the work in ensuring that questions related to the accuracy or integrity of any part of the work are appropriately investigated and resolved. All experimental procedures were approved by the Ethics Committee of Tianjin Institute of Radiation Medicine (approval number: IRM-DWLL-2019039) and were performed according to the National Institutes of Health in the Guide for the Care and Use of Laboratory Animals (NIH Publications no. 8523 , revised 1996).

Open Access Statement: This is an Open Access article distributed in accordance with the Creative Commons Attribution-NonCommercial-NoDerivs 4.0 International License (CC BY-NC-ND 4.0), which permits the noncommercial replication and distribution of the article with the strict proviso that no changes or edits are made and the original work is properly cited (including links to both the formal publication through the relevant DOI and the license). See: https://creativecommons.org/licenses/by-nc-nd/4.0/.

\section{References}

1. Wu Y, Gao Q, Zhu S, et al. Low-intensity pulsed ultrasound regulates proliferation and differentiation of neural stem cells through notch signaling pathway. Biochem Biophys Res Commun 2020;526:793-8.

2. Mortezaee K, Khanlarkhani N, Beyer C, et al. Inflammasome: Its role in traumatic brain and spinal cord injury. J Cell Physiol 2018;233:5160-9.

3. Okada $S$. The pathophysiological role of acute inflammation after spinal cord injury. Inflamm Regen 2016;36:20.

4. Lee YL, Shih K, Bao P, et al. Cytokine chemokine expression in contused rat spinal cord. Neurochem Int 2000;36:417-25.

5. Ma SF, Chen YJ, Zhang JX, et al. Adoptive transfer of M2 macrophages promotes locomotor recovery in adult rats after spinal cord injury. Brain Behav Immun 2015;45:157-70.

6. Li X, Lin S, Chen X, et al. The Prognostic Value of Serum Cytokines in Patients with Acute Ischemic Stroke. Aging Dis 2019;10:544-56.

7. Wang P, Xie ZD, Xie CN, et al. AMP-activated protein kinase-dependent induction of autophagy by erythropoietin protects against spinal cord injury in rats. CNS Neurosci Ther 2018;24:1185-95.

8. Ren Y, Young W. Managing inflammation after spinal cord injury through manipulation of macrophage function. Neural Plast 2013;2013:945034.

9. Zong S, Zeng G, Wei B, et al. Beneficial effect of interleukin-1 receptor antagonist protein on spinal cord injury recovery in the rat. Inflammation 2012;35:520-6.

10. Boato F, Rosenberger K, Nelissen S, et al. Absence of IL-1beta positively affects neurological outcome, lesion development and axonal plasticity after spinal cord injury. J Neuroinflammation 2013;10:6.

11. Mukaino M, Nakamura M, Yamada O, et al. Anti-IL-6receptor antibody promotes repair of spinal cord injury by inducing microglia-dominant inflammation. Exp Neurol 2010;224:403-14.

12. Lacroix S, Chang L, Rose-John S, et al. Delivery of hyper-interleukin-6 to the injured spinal cord increases neutrophil and macrophage infiltration and inhibits axonal growth. J Comp Neurol 2002;454:213-28.

13. Biglari B, Swing T, Child C, et al. A pilot study on temporal changes in IL-1beta and TNF-alpha serum levels after spinal cord injury: the serum level of TNF-alpha in acute SCI patients as a possible marker for neurological remission. Spinal Cord 2015;53:510-4.

14. Yang P, Wen H, Ou S, et al. IL-6 promotes regeneration and functional recovery after cortical spinal tract injury by reactivating intrinsic growth program of neurons and enhancing synapse formation. Exp Neurol 2012;236:19-27.

15. Thompson CD, Zurko JC, Hanna BF, et al. The therapeutic role of interleukin-10 after spinal cord injury. J Neurotrauma 2013;30:1311-24.

16. Khalid K, Frei J, Aboouf MA, et al. Erythropoietin Stimulates GABAergic Maturation in the Mouse 
Hippocampus. eNeuro 2021;8:ENEURO.0006-21.2021.

17. Deshmane SL, Kremlev S, Amini S, et al. Monocyte chemoattractant protein-1 (MCP-1): an overview. J Interferon Cytokine Res 2009;29:313-26.

18. Guermonprez P, Gerber-Ferder Y, Vaivode K, et al. Origin and development of classical dendritic cells. Int Rev Cell Mol Biol 2019;349:1-54.

19. McArdel SL, Terhorst C, Sharpe AH. Roles of CD48 in regulating immunity and tolerance. Clin Immunol 2016;164:10-20.

20. Jiang C, Li Z, Wu Z, et al. Integrated Bioinformatics Analysis of Hub Genes and Pathways Associated with a Compression Model of Spinal Cord Injury in Rats. Med Sci Monit 2020;26:e927107.

21. Duan HQ, Wu QL, Yao X, et al. Nafamostat mesilate attenuates inflammation and apoptosis and promotes locomotor recovery after spinal cord injury. CNS Neurosci Ther 2018;24:429-38.

22. Brennan FH, Ruitenberg MJ. Targeting acute inflammation to complement spinal cord repair. Neural Regen Res 2015;10:1596-8.

23. Beck KD, Nguyen HX, Galvan MD, et al. Quantitative analysis of cellular inflammation after traumatic spinal cord injury: evidence for a multiphasic inflammatory response in the acute to chronic environment. Brain 2010;133:433-47.

24. Jones TB. Lymphocytes and autoimmunity after spinal cord injury. Exp Neurol 2014;258:78-90.

25. Tanabe S, Yamashita T. B lymphocytes: Crucial contributors to brain development and neurological diseases. Neurosci Res 2019;139:37-41.

26. Lee YL, Shih K, Bao P, et al. Cytokine chemokine

Cite this article as: Li Q, Li B, Tao B, Zhao C, Fan B, Wang Q, Sun C, Duan H, Pang Y, Fu X, Feng S. Identification of four genes and biological characteristics associated with acute spinal cord injury in rats integrated bioinformatics analysis. Ann Transl Med 2021;9(7):570. doi: 10.21037/atm-21-603 expression in contused rat spinal cord. Neurochem Int 2000;36:417-25.

27. Zhao X, Chen C, Wei Y, et al. Novel mutations of COL4A3, COL4A4, and COL4A5 genes in Chinese patients with Alport Syndrome using next generation sequence technique. Mol Genet Genomic Med 2019;7:e653.

28. Amo-Aparicio J, Martinez-Muriana A, Sanchez-Fernandez A, et al. Neuroinflammation Quantification for Spinal Cord Injury. Curr Protoc Immunol 2018;123:e57.

29. Hayes KC, Hull TC, Delaney GA, et al. Elevated serum titers of proinflammatory cytokines and CNS autoantibodies in patients with chronic spinal cord injury. J Neurotrauma 2002;19:753-61.

30. Simon F, Floros N, Ibing W, et al. Neurotherapeutic potential of erythropoietin after ischemic injury of the central nervous system. Neural Regen Res 2019;14:1309-12.

31. Smith JR, Thackray AM, Bujdoso R. Reduced herpes simplex virus type 1 latency in Flt-3 ligand-treated mice is associated with enhanced numbers of natural killer and dendritic cells. Immunology 2001;102:352-8.

32. McArdel SL, Brown DR, Sobel RA, et al. AntiCD48 Monoclonal Antibody Attenuates Experimental Autoimmune Encephalomyelitis by Limiting the Number of Pathogenic CD4+ T Cells. J Immunol 2016;197:3038-48.

33. Yang L, Ge D, Chen X, et al. miRNA-544a Regulates the Inflammation of Spinal Cord Injury by Inhibiting the Expression of NEUROD4. Cell Physiol Biochem 2018;51:1921-31. 